\title{
Thrombocytopenia as a Marker for Diagnosis of Malaria
}

\author{
Dr. Jitendra Kumar, Dr Amit Kumar, Dr Vandana , Dr Prof Subrata Nag \\ Asst. Prof.Dept. of Medicine Narayan Med. Coll. \&HospitalSasaram Biihar \\ Asst. Prof. Dept. of PharmacologyNarayan Med Coll. \\ Asst Prof. Dept Of Physiology Narayan Med Coll. \\ HOD Anesthesiology Narayan Med. College
}

\begin{abstract}
:
Aim of the study: Malaria is major health problem in the tropics with significant high mortality and morbidity.Malaria is usually associated with reduced blood cell counts \& mild to moderate thrombocytopenia which is a common association of malaria. The cause of thrombocytopenia is poorly understood, but the immune-mediated lysis, sequestration in the spleen and dyspoietic processes in the marrow with diminished platelet production have all been postulated. This study was conducted to coorrelate thrombocytopenia in the patients suffering from acute malaria. A finding of thrombocytopenia should increase the suspicion of malaria and lead to performance of more specific tests, including multiple peripheral smears and ELISA for parasitespecific antigen.Hence we conducted this study to assess the occurrence of thrombocytopenia in hospitalized patients of malaria and to correlate its severity with the type of malaria.

Design : A total of 200 subjects were included in this study group. These patients were having high fever, intermittent in nature associated with chills and rigors, with or without splenomegaly and responded only to antimalarial drugs. Thrombocytopenia count was done by fully automated hematological analyser.

Result: Normal platelet count was observed in about $20 \%$ of cases who responded only to antimalarials while it reduced in about $80 \%$ of cases presented with fever. Thrombocyte count was 77,869 cells/ $\mu \mathrm{l}$ (mean).Thrombocytopenia was present in both (P.falciparum $>$ P. vivax) types of malaria.
\end{abstract}

\section{Introduction}

There are about 200 species in genus plasmodium about 11 species cause malaria in humans.Most cases are caused by 5 species viz. P.vivax, P.falciparum, P.malariae, P.ovale and P. Knowlesi. All except knowlesi cause malaria in humans while it affects only monkeys till now .Diagnosis of malaria is still a challenge as is not always evident by lab investigations. There are about 515 million clinical cases per year and about 6,55.000 cases died from malaria in 2010. Non-immunological as well as immunological destruction of platelets have been implicated in causing thrombocytopenia. The speculated mechanisms are coagulation disturbances, sequestration in spleen, antibody mediated platelet destruction, oxidative stress, and the role of platelets as cofactors in triggering severe malaria. Abnormalities in platelet structure and function have been described as a consequence of malaria. So in above context we have attempted to correlate low platelet with malaria.

\section{Material And Methods}

The study was done at Narayan Medical college and Hospital, Sasaram District ,Bihar,India. A total of 200 patients coming to hospital during June 2009 to June 2014 were taken, either admitted or treated on out patient basis with complaint of high grade fever which was intermittent with chills and rigors were taken for study.These patients were exclusively on antimalarials and no antibiotics was prescribed for them.Clinically few patients had splenomegaly while others just had typical malarial symptoms, while both in the study responded to antimalarials.

Exclusion criteria - Patients where localizing cause of fever could be determined ,patients with history of chronic liver disease ,bleeding disorders ,thrombocytopenia,unexplained fetal loss, purpura or history of drug intake co-trimoxazole ,thiazides ,chemotherapeutic agents were excluded.

For evaluation of platelets fully automated quantitative counter (auto- anlyser) was used for study. All cases were subjected to peripheral blood smear for malarial parasite. The cases of kala-azar ,respiratory infections, Urinary tract infection cases were excluded in study.

Repeat platelet count was done if platelet count was less than 50,000 cells/micro-liter to get rid of pseudo-thrombocytopenia which is seen due to effect of cytokines in which a large platelet gets clumped with small platelet which is interpreted as single thus giving false reading. 
III. Result

Study on patients with mean age $35 \pm 15$ year was done. Study included all cases who responded to only antimalarials with or without splenomegaly with PBS for malaria positive or negative.

\begin{tabular}{|l|l|l|l|l|}
\hline FEVER & SPLENOMEGALY & $\begin{array}{l}\text { LOW PLATELET } \\
\text { COUNT }\end{array}$ & $\begin{array}{l}\text { RESPONDED TO } \\
\text { ANTIMALARIAL }\end{array}$ & $\begin{array}{l}\text { MP SLIDE } \\
\text { POSITIVITY }\end{array}$ \\
\hline 200 & 56 & 159 & 200 & $16(\mathrm{P} . \mathrm{F}),. 36(\mathrm{P} . \mathrm{V})$. \\
\hline & $28 \%$ & $79.5 \%$ & $100 \%$ & $8 \% \quad 18 \%$ \\
\hline
\end{tabular}

Total samples- 200

Mean value platelet no.- 77,869 cells/ $\mu \mathrm{l}$

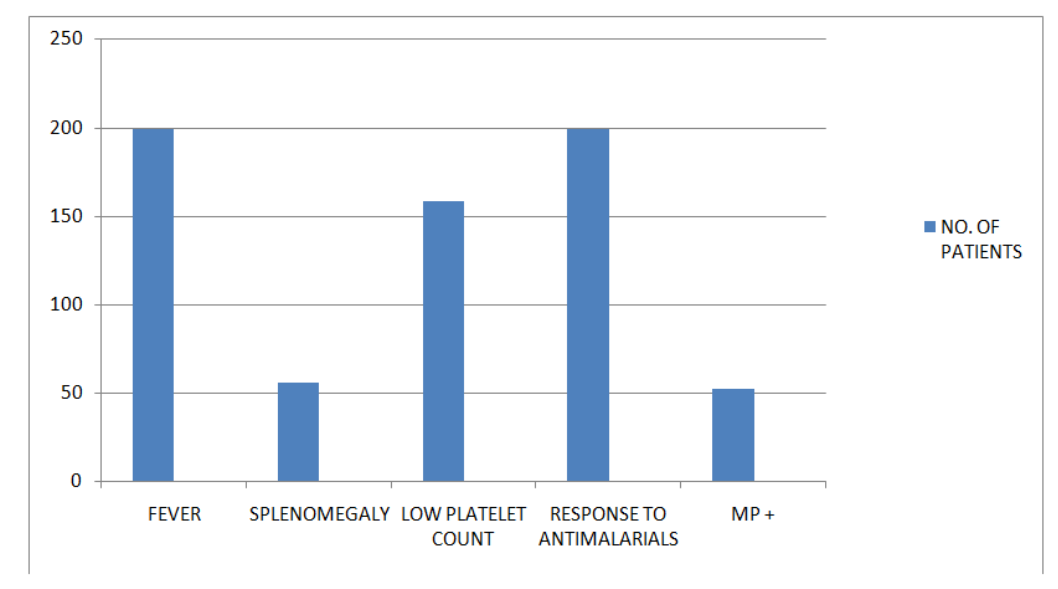

IV. Discussion

Malaria is a major health issue for people residing in tropical and sub-tropical areas. Mortality and morbidity is mainly due to the delayed diagnosis and treatment of this potentially treatable disease. The classical pattern of disease when there is cyclic fever coinciding with the release of schizonts in the blood stream is seen in only a small percentage of cases. As there is no clinical localizing sign in malaria, therefore, it is easily confused with other diseases like enteric fever, Dengue fever or viral illness. In this study, thrombocytopenia was taken as the haematological parameter. It is a general consensus that thrombocytopenia is very common in malaria. In this study there were three important findings -

1. Thrombocytopenia was a common laboratory feature;

2. Both the Plasmodium species (P.falciparum $>$ P.vivax) were associated with it.

3. Nearly $76 \%$ of patients with suspected malaria developed within 3-5 days of infection which returned to normal in 5-7 days of giving anti-malarial treatment.

Exact mechanism of thrombocytopenia in malaria is unknown. Fajardo and Tallent demonstrated $P$. vivax within platelets and suggested a direct lytic effect of the parasite on the platelets. Both non-immunological destruction as well as immune mechanism involving specific platelet associated IgG antibodies that bind directly to malarial antigen in the platelets have been recently reported to play a role in the lysis of platelets. Oxidative stress damage of platelets has also been implicated in the etiopathogenesis based on the finding of low levels of platelet superoxide-dismutase and glutathione peroxidase activity and high platelet lipid peroxidation levels in malaria patients, when compared to those of healthy subjects. Decreased thrombopoiesis has been ruled out, because platelet forming megakaryocytes in the marrow are usually normal or increased. A good tolerance of low platelet count is well-known in malaria.

Two types of platelet dysfunction are encountered in malaria,platelet hyperactivity and hypoactivity. Hyperactivity results from various aggrevating agents like immune complexes ,platelet surface contact with infected RBCs and damage to endothelial cells.Injured platelet undergoes intravascular hemolysis and releases cellular contents of the platelets that activate intrinsic coagulation cascade, as contributed to DIC. The hyperactive platelets may enhance hemostatic responses and that is why bleeding episodes are very rare in acute malarial infections, despite significant thrombocytopenia.

Thrombocytopenia per se cannot differentiate between the types of malaria but according to statistics and previous studies it has been more linked to P.falciparum malaria than P.vivax. However even severe P.vivax can give rise to thrombocytopenia. 


\section{Conclusion}

Thrombocytopenia is common occurrence in malaria as can be used as a reliable diagnostic marker for supporting malaria in presence of PBS negative for malarial parasite.

\section{References}

[1]. Kakar A, Bhoi S, Prakash V, Kakar S. Profound thrombocytopenia in Plasmodium vivax malaria. Diagn Microbiol Infect Dis 1999;35:243-4

[2]. S. S. Jamal Khan, F. R. Khan, M. Usman, and S. Zahid, "Malaria can lead to thrombocytopenia," Rawal Medical Journal, vol. 33, no. 2, pp. 183-185, 2008

[3]. T. S. Faseela, R. A. Roche, K. B. Anita, C. S. Malli, and Y. Rai, "Diagnostic value of platelet count in malaria," Journal of Clinical and Diagnostic Research, vol. 5, no. 3, pp. 464-466, 2011

[4]. V. Bhatia and J. Bhatia, "Severe thrombocytopenia with bleeding manifestations in two children secondary to Plasmodium vivax," Platelets, vol. 21, no. 4, pp. 307-309, 2010

[5]. R. Harish and S. Gupta, "Plasmodium vivax malaria presenting with severe thrombocytopenia, cerebral complications and hydrocephalus," Indian Journal of Pediatrics, vol. 76, no. 5, pp. 551-552, 2009.

[6]. World Health Organization. (2011) World malaria report 2011. Geneva: World Health Organization. xii, 246 p. p.

[7]. Tanwar GS, Khatri PC, Chahar CK, Sengar GS, Kochar A, et al. (2012) Thrombocytopenia in childhood malaria with special reference to $P$. vivax monoinfection: A study from Bikaner (Northwestern India). Platelets 23: 211-216. doi: $10.3109 / 09537104.2011 .607520$

[8]. Klein E, Ronez E (2012) Peripheral hemophagocytosis in malaria infection. Blood 119: 910. doi: 10.1182/blood-2011-02-336420

[9]. Saravu K, Docherla M, Vasudev A, Shastry BA (2011) Thrombocytopenia in vivax and falciparum malaria: an observational study of 131 patients in Karnataka, India. Ann Trop Med Parasitol 105: 593-598. doi: 10.1179/2047773211y.0000000013

[10]. Andrade BB, Barral-Netto M (2011) Biomarkers for susceptibility to infection and disease severity in human malaria. Mem Inst Oswaldo Cruz 106 Suppl 170-78. doi: 10.1590/s0074-02762011000900009

[11]. Colonel KM, Bhika RD, Khalid S, Khalique-ur-Rehman S, Syes ZA. Severe thrombocytopenia and prolonged bleeding time in patients with malaria (a clinical study of 162 malaria cases). World Appl Sci J 2010;9:484-8. ^ 